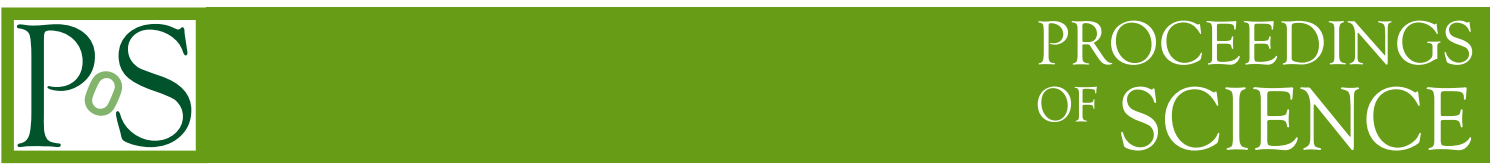

\title{
Description of $Z+b \bar{b}$ with 4 flavour and 5 flavour transverse momentum dependent PDFs
}

\author{
S. Taheri Monfared, ${ }^{a, *}$ H. Jung ${ }^{a}$ and F. Hautmann ${ }^{b, c}$ \\ ${ }^{a}$ DESY, Hamburg, Germany \\ ${ }^{b} R A L$, Chilton OX11 OQX and University of Oxford, OX1 3NP \\ ${ }^{c}$ Elementary Particle Physics, University of Antwerp, B 2020 Antwerp \\ E-mail: sara.taheri.monfared@desy.de, hannes.jung@desy.de, \\ hautmann@thphys.ox.ac.uk
}

We generate 4 flavour version of the NLO-PB PDFs by performing a fit to deep inelastic scattering (DIS) experimental data. 4 flavour Transverse Momentum Dependent (TMD) parton distribution functions (PDFs) calculated with the Parton Branching (PB) method are applied to predict two LHC processes: the transverse momentum spectrum of $Z$ boson and the azimuthal angular separation $\Delta \phi_{b b}$ between two $b$ jets in proton-proton collisions at a center-of-mass energy of $8 \mathrm{TeV}$.

40th International Conference on High Energy physics - ICHEP2020

July 28 - August 6, 2020

Prague, Czech Republic (virtual meeting)

\footnotetext{
${ }^{*}$ Speaker
} 


\section{Introduction}

We discus new aspects of the Parton Branching evolution method developed in [1, 2]. PDF sets obtained for different number of active quark flavours. We determine collinear and transverse momentum dependent (TMD) PDFs for 4 active flavours and show an application of these TMD densities to the calculation of $Z+b$ production [3] at th Large Hadron Collider (LHC).

\section{Method description}

In the PB approach we can calculate the complete evolution of the parton density which at the same time gives full information of the kinematic of the evolution process. Within this method, the parton shower is strictly tied to the parton density which includes soft-gluon resummation to all orders in $\alpha_{s}$. Soft gluon emission and transverse momentum recoils are expressed by introducing the soft gluon resolution scale $z_{M}$. The evolution without resolvable branching from $\mu_{0}^{2}$ to $\mu^{2}$ is treated via Sudakov form factors

$$
\Delta_{a}\left(\mu^{2}, \mu_{0}^{2}\right)=\exp \left[-\sum_{b} \int_{\mu_{0}^{2}}^{\mu^{2}} \frac{d \mu^{\prime 2}}{\mu^{\prime 2}} \int_{0}^{z_{M}} d z z P_{b a}^{(R)}\left(z, \alpha_{s}\right)\right],
$$

Here, $z_{M}$ separates resolvable and non-resolvable branchings, $z$ is the longitudinal momentum fraction, $\alpha_{s}$ is the strong coupling and $P_{b a}^{(R)}$ represent real emission splitting functions from flavour $a$ to $b$. In this approach the TMD evolution equations are written as

$$
\begin{aligned}
\mathcal{A}_{a}\left(x, \mathbf{k}, \mu^{2}\right) & =\Delta_{a}\left(\mu^{2}\right) \mathcal{A}_{a}\left(x, \mathbf{k}, \mu_{0}^{2}\right)+\sum_{b} \int \frac{d^{2} \mathbf{q}^{\prime}}{\pi \mathbf{q}^{\prime 2}} \frac{\Delta_{a}\left(\mu^{2}\right)}{\Delta_{a}\left(\mathbf{q}^{\prime 2}\right)} \Theta\left(\mu^{2}-\mathbf{q}^{\prime 2}\right) \Theta\left(\mathbf{q}^{\prime 2}-\mu_{0}^{2}\right) \\
& \times \int_{x}^{z_{M}} \frac{d z}{z} P_{a b}^{(R)}\left(\alpha_{\mathrm{s}}, z\right) \mathcal{A}_{b}\left(\frac{x}{z}, \mathbf{k}+(1-z) \mathbf{q}^{\prime}, \mathbf{q}^{\prime 2}\right)
\end{aligned}
$$

where $\mathcal{A}_{a}\left(x, \mathbf{k}, \mu^{2}\right)$ is the TMD distribution of flavour $a$, carring the longitudinal momentum fraction $x$ of the hadron's momentum and transverse momentum $\mathbf{k}$ at the evolution scale $\mu^{2}$. The first set of variable flavour number scheme (VFLNS) TMDs evolved with NLO DGLAP splitting functions determined from a fit to HERA I+II precision measurements [4] are described in Ref. [5]. The PB method has been implemented in the xFitter package [6] to allow fits to be made to cross section measurements.

\section{4 flavour scheme parton distributions}

We determined collinear and TMD PDFs in 4 flavour (4FLNS) scheme from a fit to HERA combined data in the ranges $3.5<Q^{2}<50000 \mathrm{GeV}^{2}$ and $4.10^{-5}<x<0.65$. The heavy quark is treated massless in the evolution but the number of flavours changes according to the mass threshold [7]. In the 4FLNS the bottom quark mass is set to infinity. We produced the 4 flavour partons by performing a fit within this scheme. The fit is performed using the open-source fitting platform xFitter package with the same functional forms for initial densities as 5FLNS. Our procedure is different from the other PDF sets (e.g MMHT [8], CT14 [9]) who just obtained 4 flavour set by using the input PDFs obtained in the 5 flavour together with the evolution of the bottom quark off. 
In 4FLNS, it is essential to choose a consistent value of strong coupling constant $\alpha_{S}$. In our default NLO fit [5] we put $\alpha_{S}^{(5)}\left(m_{Z}^{2}\right)=0.118$. When obtaining the 4FLNS collinear PDFs and TMDs, the contribution of the bottom quark decouples and the coupling itself does not include the $b$ quark as an active flavour. On this basis we define the TMD-PDFs using this 4 flavour definition of the coupling only in the evolution. Restricting ourself to a maximum of four active flavours gives $\alpha_{S}^{(4)}\left(m_{Z}^{2}\right)=0.1128$. The mass of the charm quark is set $m_{c}=1.47 \mathrm{GeV}$. We obtain 4FLNS collinear and TMD PDF sets at NLO performing a fit on HERA data [4]. In Fig. 1 the 4 flavour and 5 flavour collinear parton densitiesas in the PB-TMD set 2 are shown. The argument in the strong coupling is taken to be the transverse momentum $q_{t}$. This choice yields good $\chi^{2}$ value for the fit to DIS measurements. We reach $\chi^{2} / \operatorname{dof}=1.254$ which is slightly bigger than 5 flavour results $\left(\chi^{2} /\right.$ dof $\left.=1.21\right)$.
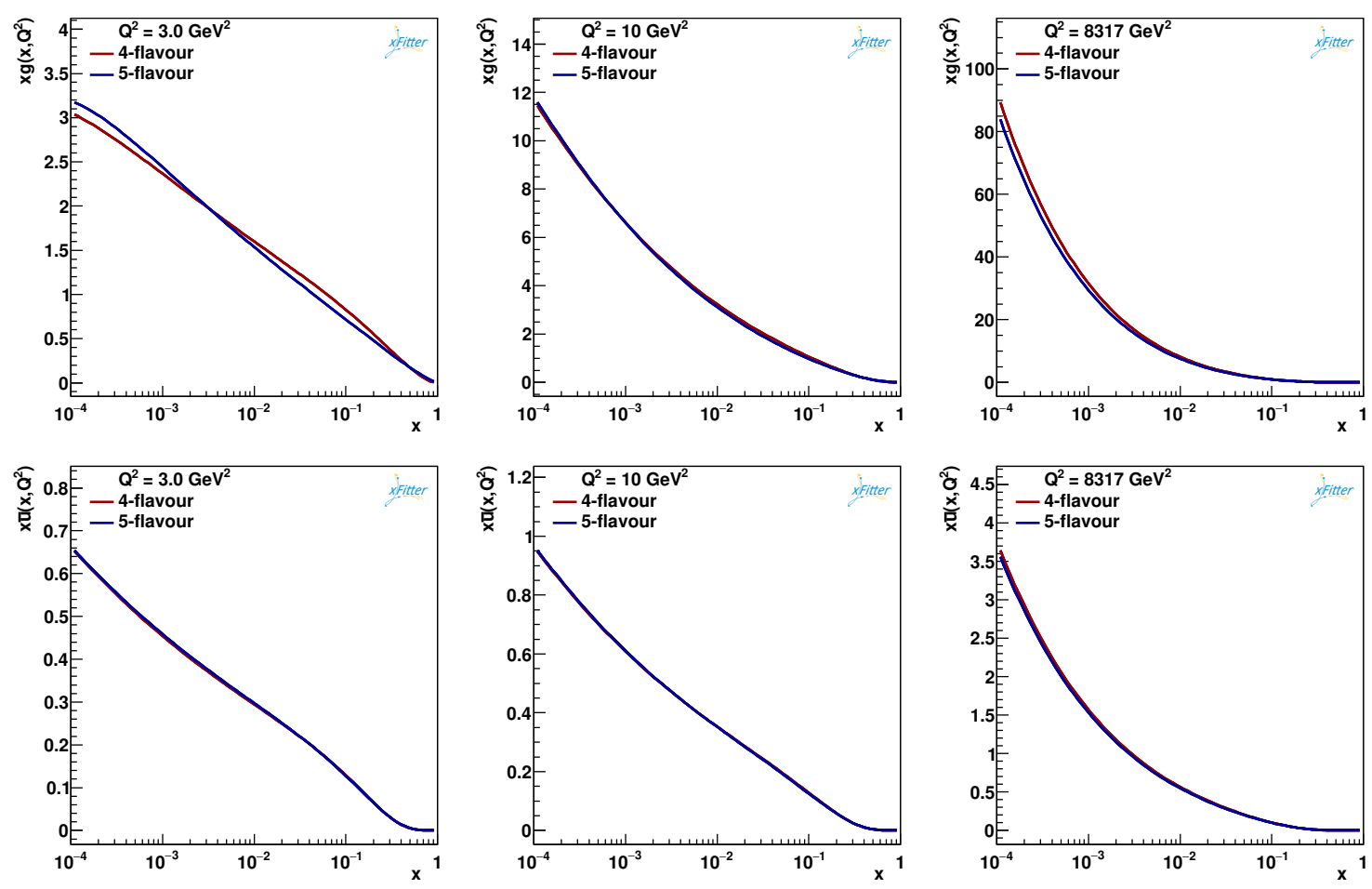

Figure 1: The gluon and $\bar{u}$-type 4 flavour and 5 flavour densties for different valuses of $\mu^{2}$.

In Fig. 2, TMD PDFs are shown as a function of $k_{t}$ with 4 and 5 active flavours for the $\bar{u}$ (Fig. 2.a), charm (Fig. 2.b), bottom and gluon (Fig. 2.c). The $b$ quark only exists in 5FLNS. At small $k_{t}$ essentially the first term in Eq.2 contributes without any resolvable branching. Here, transverse momentum distributions based on 4 FLNS and 5 FLNS are different because they have different starting distributions. At large $k_{t}$ several branching may have occurred and the differences are washed out due to having more splittings.

\section{Calculation of the $Z+b$ jets production in 4 flavour and 5 flavour}

For the calculation of the $Z+b(b)$ cross section, two approaches of the 4FLNS and the 5FLNS are applied. In the 4FLNS only the four lightest quarks $(u, d, c$ and $s)$ are present in the initial state 


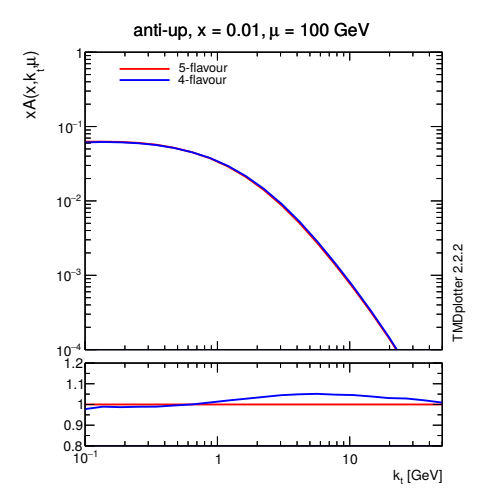

(a) $\bar{u}$

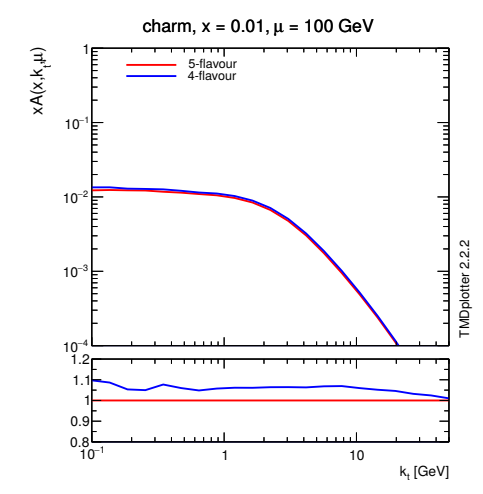

(b) charm

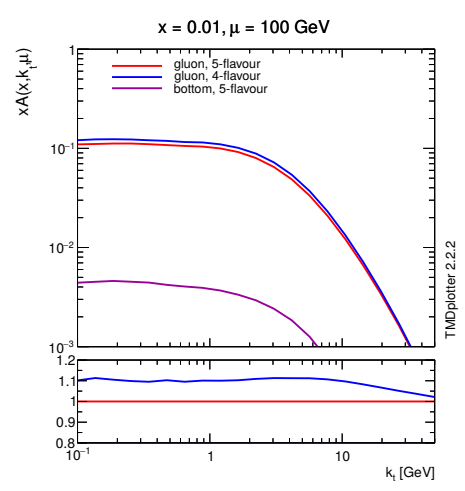

(c) bottom and gluon

Figure 2: The $\bar{u}$ (a), charm (b), bottom and gluon (c) 4 flavour and 5 flavour TMDs as a function of $k_{t}$ for $x=0.01 \mathrm{GeV}$ at $\mu=100$.

and a final state $b$ quarks are generated from the gluon splitting, as described by the QCD matrix element (ME). On the other hand, in 5FLNS, the $b$ quark has a non-zero parton density above the $m_{b}$ threshold.

We use these 4 flavour and 5 flavour PB-TMDs to predict the $Z+b \bar{b}$ production in pp collisions, applying NLO collinear calculations of the hard scattering process obtained with the MAdGraph5_AMC@NLO (labelled MC@NLO) [10] framework. As in [11], the PB-TMDs are applied to generate transverse momenta of the incoming partons. We consider Herwig6 [12] subtraction terms in MC@NLO, motivated by angular ordering in PB evolution. The LHE files generated by MC@NLO generates are read by parton shower event generators CASCADE package $[13,14]$ (version 3.0.X) . We presented an example of Feynman diagram for the $Z+b$ process in the 5 FLNS and $Z+b b$ in the 4 FLNS in Fig3.a and Fig3.b.

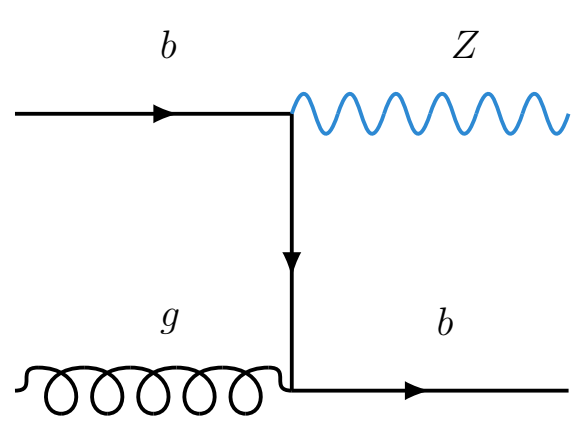

(a) $Z+b 5$ FLNS diagram

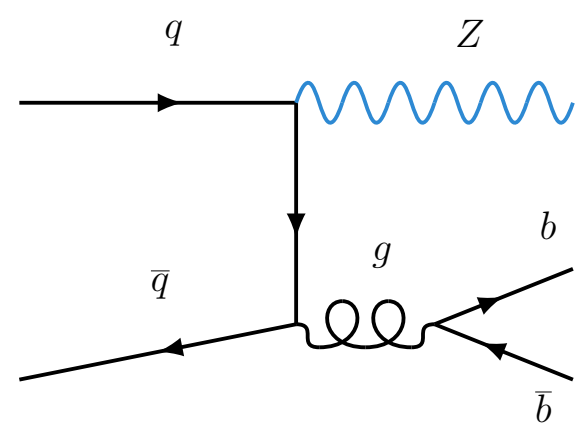

(b) $Z+b b 4$ FLNS diagram

Figure 3: The LO Feynman diagrams for $Z+b$ and $Z+b b$ production in 5 FLNS (a) and 4 FLNS (b). 


\section{Application to $Z$ boson production at the LHC}

Accurate $Z+b$ jet measurements at the $\sqrt{s}=8 \mathrm{TeV}$ [1] can provide a useful ground for testing the differences between the 4 FLNS and the 5 FLNS implementation of perturbative QCD predictions. We employ the PB formulation of TMD evolution set out in [5] and calculate 4 flavour TMD and collinear PDFs to describe the production of $Z+\geq 2 b$ jets at NLO within 4 FLNS and 5 FLNS. The PB method has the advantage that parton showers can be included following exactly the evolution of the TMD parton density. The transverse momentum of the initial state partons is calculated according to the TMDs as implemented in the CASCADE package (version 3.0.X) where events are produced in HEPMC [15] format for further processing with Rivet [16].

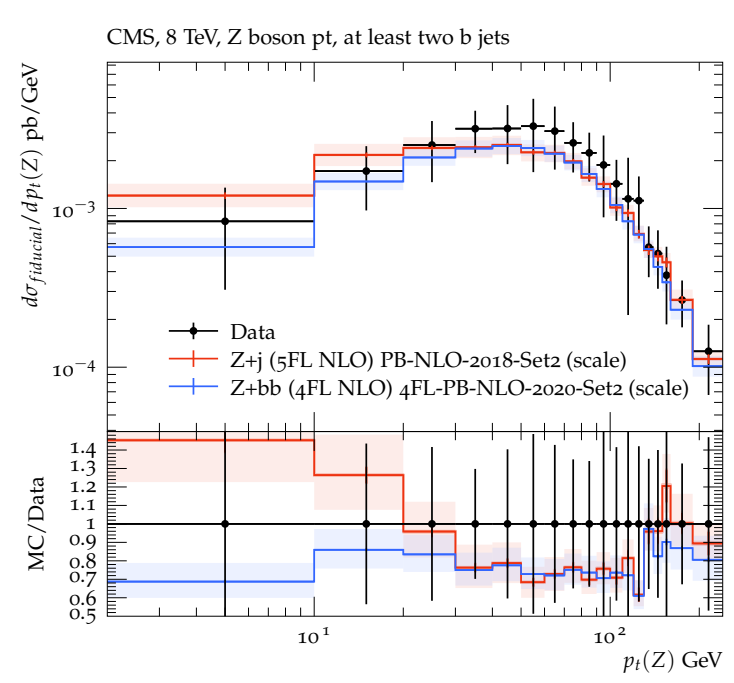

(a)

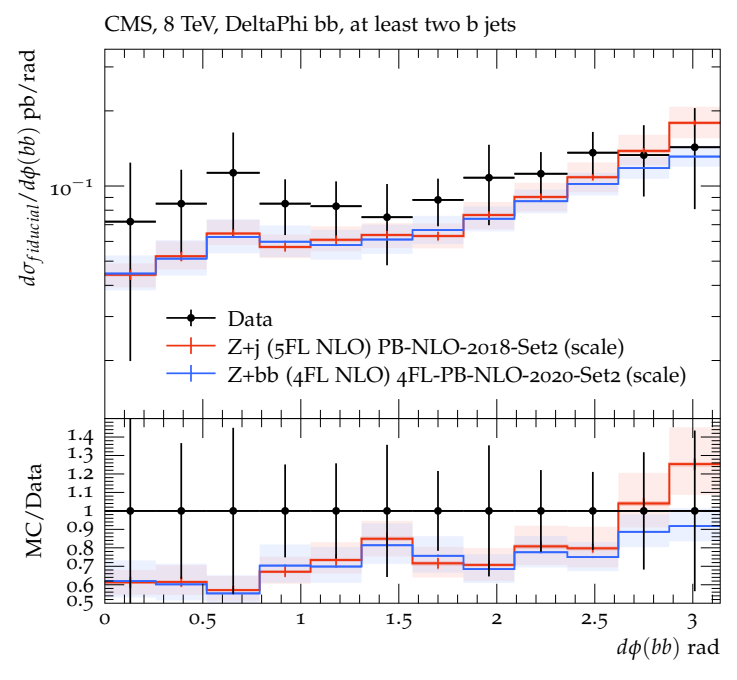

(b)

Figure 4: Differential cross section for $Z+\geq 2 b$ jets production as a function transverse momentum of the $Z$ boson $p_{t}$ (a) and the azimuthal angular separation $\Delta \phi_{b b}$ between the directions of the two $b$ jets in the transverse plane $(b)$.

We calculated $Z+b$ production with 4 and 5 flavour PB-TMDs and compare our calculation to measurements of CMS [3] at $8 \mathrm{TeV}$. In Fig. 4.a we show the prediction of the transverse momentum of the $Z$-boson and at least two $b$ jets and in Fig. 4.b the azimuthal angular separation $\Delta \phi_{b b}$ between the two $b$ jets. The 4 flavour and 5 flavour calculations do agree and can describe the measurements well. We conclude, that the TMD parton shower together with TMD parton densities obtained in the 5 flavour scheme give consistent results with the predictions obtained in the 4 flavour scheme. This gives confidence that both the heavy flavour PB TMDs as well as the heavy flavour TMD parton shower as implemented in CASCADE3 provides a reliable description.

\section{References}

[1] F. Hautmann et al., "Soft-gluon resolution scale in QCD evolution equations", Phys. Lett. B772 (2017) 446, arXiv: 1704.01757. 
[2] F. Hautmann et al., "Collinear and TMD quark and gluon densities from Parton Branching solution of QCD evolution equations”, JHEP 01 (2018) 070, arXiv: 1708. 03279.

[3] V. Khachatryan et al. [CMS], Eur. Phys. J. C 77 (2017) no.11, 751, arXiv: 1611.06507.

[4] ZEUS, H1 Collaboration, Eur. Phys. J. C75 (2015), no. 12, 580, arXiv: 1506.06042.

[5] A. Bermudez Martinez, P. Connor, H. Jung, A. Lelek, R. Žlebčík, F. Hautmann and V. Radescu, Phys. Rev. D 99 (2019) no.7, 074008, arXiv: 1804 . 11152.

[6] S. Alekhin et al., Eur. Phys. J. C75 (2015), no. 7, 304, arXiv: 1410.4412.

[7] A. Kusina, F. I. Olness, I. Schienbein, T. Jezo, K. Kovarik, T. Stavreva and J. Y. Yu, Phys. Rev. D 88 (2013) no.7, 074032, arXiv: 1306.6553.

[8] L. A. Harland-Lang, A. D. Martin, P. Motylinski and R. S. Thorne, Eur. Phys. J. C 76 (2016) no.1, 10, arXiv: 1510.02332.

[9] S. Dulat et al., Phys. Rev. D93 (2016), no. 3, 033006, arXiv: 1506. 07443.

[10] J. Alwall, R. Frederix, S. Frixione, V. Hirschi, F. Maltoni et al. , JHEP 1407 (2014) 079 arXiv: 1405.0301.

[11] A. Bermudez Martinez et al.,Phys. Rev. D100 (2019) 074027 arXiv: 1906.00919.

[12] G. Corcella, I. Knowles, G. Marchesini, S. Moretti, K. Odagiri et al., arXiv: 0210213.

[13] H. Jung et al., Eur.Phys.J. C70 (2010) 1237, arXiv: 1008.0152.

[14] H. Jung, Comput. Phys. Commun. 143 (2002) 100, arXiv: 0109102.

[15] M. Dobbs and J. B. Hansen, Comput. Phys. Commun. 134 (2001) 41.

[16] A. Buckley et al. Comput. Phys. Commun. 184 (2013) 2803-2819, arXiv: 1003. 0694. 